University of Nebraska - Lincoln

DigitalCommons@University of Nebraska - Lincoln

John R. Hardy Papers

Research Papers in Physics and Astronomy

1997

\title{
Raman-Scattering Study of the Order-Disorder Phase Transition in KSCN
}

\author{
R. Li \\ University of Nebraska - Lincoln \\ John R. Hardy \\ University of Nebraska - Lincoln
}

Follow this and additional works at: https://digitalcommons.unl.edu/physicshardy

Part of the Physics Commons

Li, R. and Hardy, John R., "Raman-Scattering Study of the Order-Disorder Phase Transition in KSCN" (1997). John R. Hardy Papers. 71.

https://digitalcommons.unl.edu/physicshardy/71

This Article is brought to you for free and open access by the Research Papers in Physics and Astronomy at DigitalCommons@University of Nebraska - Lincoln. It has been accepted for inclusion in John R. Hardy Papers by an authorized administrator of DigitalCommons@University of Nebraska - Lincoln. 


\title{
Raman-scattering study of the order-disorder phase transition in $\mathrm{KSCN}$
}

\author{
R. Li and J. R. Hardy \\ Department of Physics and Astronomy and Center for Electro-optics, University of Nebraska-Lincoln, Lincoln, Nebraska 68588-0111
}

(Received 9 May 1997)

\begin{abstract}
Raman measurements were performed from room temperature to above the phase transition at $413 \mathrm{~K}$ on KSCN crystals. The internal mode spectra reveal a sideband above the internal $\mathrm{CN}$ stretching vibration which shows softening in frequency approaching the phase transition, while the $\mathrm{CN}$ mode shifts toward higher frequencies. Given that the sideband is a two-phonon process, involving an off-zone-center CN stretching mode and a lattice phonon, the substantial softening in its frequency shift indicates the possibility of an off-zone-center lattice instability related to the order-disorder phase transition. An anomalous temperature dependence was also found for the intensity of both the $\mathrm{CN}$ mode and the sideband. The relatively high intensity of the sideband was analyzed in terms of a substantial Fermi resonance coupling between the internal mode and the sideband. [S0163-1829(97)07546-2]
\end{abstract}

KSCN and other thiocynates are of interest as systems containing triatomic linear $\mathrm{SCN}^{-}$units which show orientational disorder at elevated temperatures. ${ }^{1,2} \mathrm{KSCN}$ crystallizes at an ordered orthorhombic structure of $D_{2 h}^{11}-\mathrm{Pbcm}$ symmetry at room temperature. There are four KSCN molecules in the primitive cell in this phase and the four linear $\mathrm{SCN}^{-}$ clusters lie on the [001] plane. As the temperature is raised, the triatomic $\mathrm{SCN}^{-}$ions are thermally activated to be able to flip between (110) and (1-10) directions within the [001] plane, which correspond to two minima in the orientational potential surface. When the temperature is increased to $T_{c}=413 \mathrm{~K}$, the $\mathrm{SCN}^{-}$ions become able to reorient between these two minima, like the $\mathrm{NO}_{2}^{-}$ions in $\mathrm{NaNO}_{2}$ above the high-temperature phase transition, ${ }^{4}$ and the system undergoes a $\lambda$-type phase transition into a disordered tetragonal phase of $D_{4 h}^{18}-I 4 / \mathrm{mcm}$ symmetry (two units per primitive cell). The tetragonal symmetry of the high-temperature phase rests on, in addition to the equivalence of $\mathbf{a}$ and $\mathbf{b}$ lattice constants, a random distribution of the $\mathrm{SCN}^{-}$ions between the two orientations of equal minimum energy. ${ }^{1}$

There have been several spectroscopic investigations of the order-disorder transition in $R \mathrm{SCN}$ systems (where $R$ is either $\mathrm{K}, \mathrm{Rb}$, or $\mathrm{Cs}$ ). ${ }^{3,5-10}$ These studies focused on the temperature dependence of the $\Gamma$-point vibrations and have shown that the phase transition in such systems is accompanied by anomalies in the vibrational spectra. ${ }^{3,8-10}$ The major concerns of these studies have been to address lattice effects on the transition, to determine the potential barrier for the reorientation of $\mathrm{SCN}^{-}$ions, and to extract the critical exponent of the order parameter from the phonon data. However, other aspects, such as the possibility of an off-zone-center instability and the relation of the internal vibrations to the order-disorder phase transition, also deserve attention.

An off-zone-center phonon anomaly was discussed in the contest of the order-disorder phase transition in systems such as $\mathrm{NH}_{4} \mathrm{Br}^{11,12}$ For KSCN such an instability was suggested in a recent review ${ }^{1}$ from the form of the order parameter, but no more direct proof of this has been reported. However, the SCN bending and the CS stretching modes, at 480 and 750 $\mathrm{cm}^{-1,9}$ respectively, exhibit frequency softening approach- ing $T_{c},{ }^{10}$ which indicates substantial coupling to the lattice motions and significant involvement in the order-disorder phase transition. Similarly, the $\mathrm{CN}$ stretching vibration at $2060 \mathrm{~cm}^{-1}$ (Ref. 10) shows hardening around the transition.

In this work, we will present a Raman study of singlecrystal KSCN with emphasis on the lattice instability and anharmonicity. The temperature evolution of the spectra of the $\mathrm{CN}$ internal mode region was followed very closely, revealing anomalous changes of both the $\mathrm{CN}$ stretching mode and a sideband above it. The most interesting feature is that the sideband exhibits a substantial softening of $\sim 12 \mathrm{~cm}^{-1}$ between $300 \mathrm{~K}$ and the phase transition. Assuming that the sideband is a two-phonon process involving an off-zonecenter lattice phonon and an internal mode with the opposite wave vector, this softening indicates a possible off-zone center instability of the lattice accompanying the phase transition. Also, the relatively high intensity of the sideband suggests a substantial anharmonic coupling between the $\mathrm{CN}$ internal mode and the lattice vibration. As we should show, a simple two coupled oscillator model gives a nearly linear temperature dependence for this anharmonic coupling.

Single crystals of KSCN were grown by slow evaporation of a saturated acetone solution of commercially available KSCN powder. ${ }^{13}$ The crystals obtained were transparent and colorless. They are of cuboid shape with mirrorlike surfaces, and typically $2 \times 2 \times 4 \mathrm{~mm}$ in size. As pointed out in Ref. 13, the long dimension direction of a crystal prepared this way indicates the $c$ axis, and each rectangular surface is parallel either to the $[110]$ or to the $[1 \overline{1} 0]$ plane. Assuming this is the case for the orientation of our crystals, we characterized our sample by measuring Raman spectra in various configurations. The spectra obtained agree very well with those of the same symmetry for KSCN reported in the literature. ${ }^{6}$

The Raman measurements were performed with a double monochromator, standard photon counting electronics, and a Spectra Physics $\mathrm{Ar}^{+}$laser as excitation source. A homedesigned heating device was built for the high-temperature measurements. Because of the size of the crystals and their mirrorlike surfaces, no polishing was done on the measured samples. The spectra were recorded between $300 \mathrm{~K}$ and 


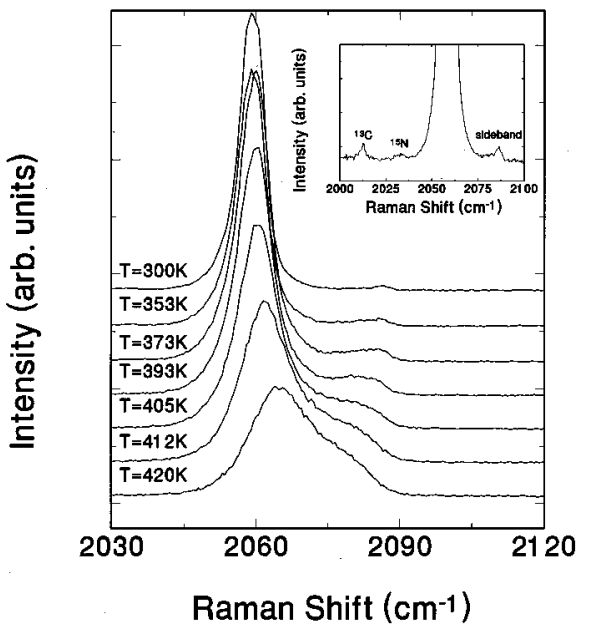

FIG. 1. Series of $A_{g}$ Raman spectra taken at $T=300,353,373$, $393,405,412$, and $420 \mathrm{~K}$, respectively. The curves are displaced upwards evenly along the intensity axis for clarity. Note the change of the line shape of the sideband with temperature. The inset is an enlargement of the weak peaks of the room-temperature spectrum for clarity.

above $T_{c}$ in the $90^{\circ}$ geometry with the $514.5 \mathrm{~nm}$ laser line. The temperature reading from the temperature sensor was calibrated using the anti-Stokes and Stokes intensities, and temperatures quoted below are the corrected values.

In this work we will focus on the $\mathrm{CN}$ internal mode region only. The remainder of the spectra will be discussed elsewhere. According to group-theory analysis, ${ }^{3,5}$ all three internal modes can appear in both the $A_{g}$ and $B_{1 g}$ spectra of the crystal. The room-temperature spectra of various geometries have been well documented in literature, here we present only $\left(X^{\prime}, Y^{\prime}\right)$, where $X^{\prime}$ and $Y^{\prime}$ designate the ab diagonal and its perpendicular, geometry spectra which select the $A_{g}$ symmetry component. Figure 1 shows a series of spectra taken at various temperatures between 300 and 420 $\mathrm{K}$.

The strongest peak at $2059 \mathrm{~cm}^{-1}$ in the room-temperature spectrum is the $\mathrm{CN}$ stretching mode. In addition, weak peaks are observed at 2013, 2032, and $2086 \mathrm{~cm}^{-1}$. The first two peaks are due to the ${ }^{13} \mathrm{C}$ and ${ }^{15} \mathrm{~N}$ isotopes. Since, as already reported, ${ }^{3,7}$ the vibrational spectra of the KSCN are rich in multiphonon processes, ${ }^{6,7}$ the peak at $2086 \mathrm{~cm}^{-1}$ was reported in Ref. 7 among other multiphonon features and was considered to be a two-phonon scattering process involving the $\mathrm{CN}$ internal mode and a lattice phonon. Usually a two phonon process consists of phonons with wave vectors from some special points of the Brillouin zone where the density of state is high. ${ }^{3}$ Such points, called critical points, can occur at the center or the edges or in some case along the highsymmetry lines of the Brillouin zone. However, in the present case, assuming that the two-phonon argument is valid, the zone center possibility can be ruled out, since there is no match between the frequency of any of the lattice phonons $^{14}$ and the frequency difference between the $\mathrm{CN}$ mode and the sideband, which is about $27 \mathrm{~cm}^{-1}$ at room temperature. Thus, the sideband must involve contributions from the points away from the zone center. However, since there is no experimental information on the phonondispersion curves of $\mathrm{KSCN}$, it is hard to tell if the sideband is
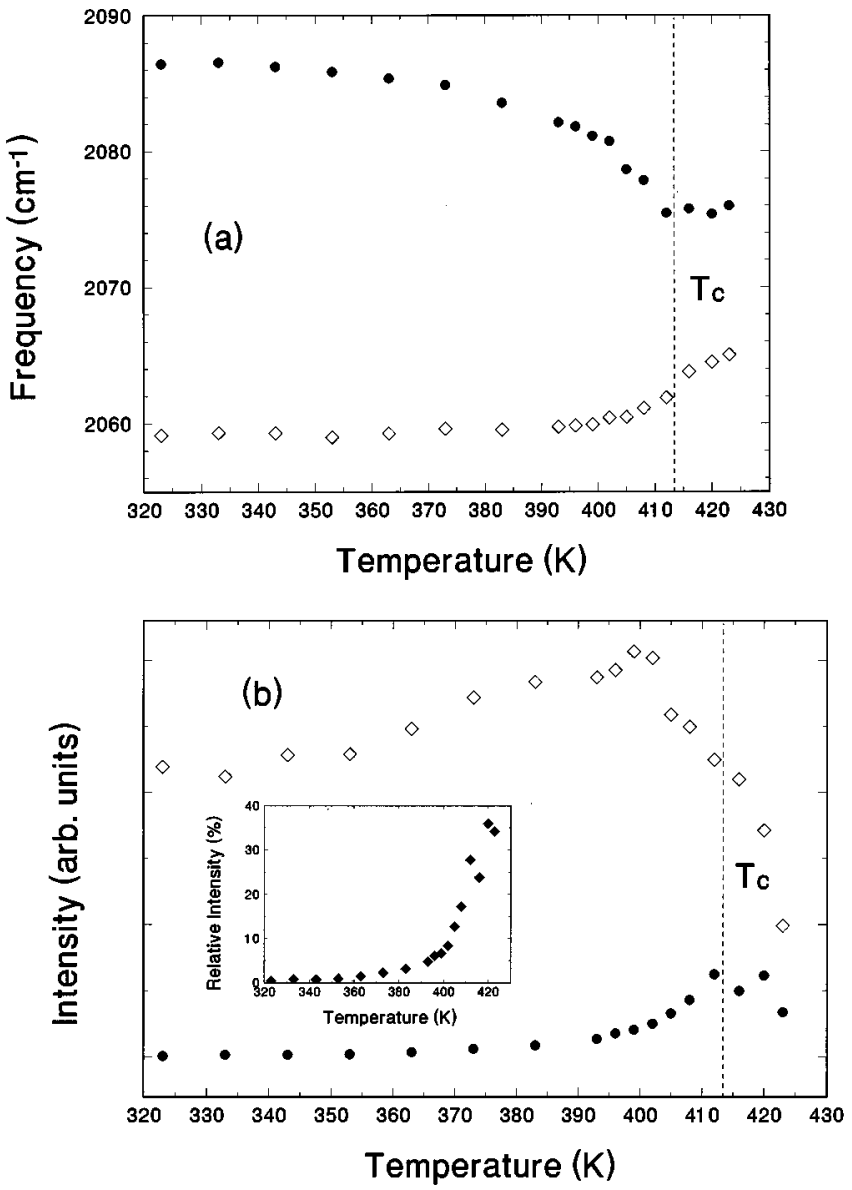

FIG. 2. The temperature dependence of the frequency (a) and the integrated intensity (b) of the CN internal mode $(\diamond)$ and the sideband ( ). The softening of the sideband and the hardening of the $\mathrm{CN}$ mode are clearly seen in (a). The inset in (b) shows the change of the ratio of the intensity of the sideband to that of the $\mathrm{CN}$ mode with temperature.

a difference or a combination process. In addition, one has to note that usually a two-phonon process is rather weak because its intensity depends on the second derivative of the polarizability. ${ }^{15}$ This relatively high intensity of the sideband, especially at higher temperatures, we ascribe to anharmonic coupling to the neighboring $\mathrm{CN}$ mode as discussed below.

As the temperature is raised, one can see very marked changes in the two peaks as shown in Fig. 1, specifically: (1) the $\mathrm{CN}$ mode becomes less intense at higher temperature, while the sideband becomes stronger, (2) the two peaks become closer as the temperature rises, as the $\mathrm{CN}$ mode is shifting to higher frequency, while the sideband is softening, (3) while the line shape of the $\mathrm{CN}$ mode stays basically Lorentzian over the whole temperature range, the profile of the sideband changes with temperature: being quite asymmetric at room temperature and becoming symmetric at higher temperatures, (4) both peaks broaden markedly with increasing temperature.

In order to understand these changes, we fit the spectra with two Lorentzians plus a constant background, recognizing that a single Lorentzian may not be fully adequate for the sideband at the lower temperatures. In Figs. 2(a) and 2(b) we show the fitted frequencies and the integrated intensities, re- 
spectively. The frequency of the $\mathrm{CN}$ mode does not seem to be influenced by temperature until about $400 \mathrm{~K}$, then, it begins to increase, reaching $2064 \mathrm{~cm}^{-1}$ at $T_{c}$. This increase continues above $T_{c}$ but with a reduced slope. On the other hand, the sideband starts to decrease in frequency just above room temperature, and softens by about $12 \mathrm{~cm}^{-1}$ at $T_{c}$. After the phase transition, the frequency of the sideband stays almost a constant. As regards the intensity change of the two peaks [Fig. 2(b)], one interesting feature is that the intensity of the CN mode, after increasing linearly from room temperature, begins to fall above $400 \mathrm{~K}$. This is almost the same temperature at which the hardening of the frequency of the $\mathrm{CN}$ mode begins. This may indicate a precursor anomaly beginning about $15 \mathrm{~K}$ below $T_{c}$. The intensity of the sideband shows a continuous nonlinear increase toward $T_{c}$, from less than $1 \%$ of that of the $\mathrm{CN}$ mode at room temperature to about $30 \%$ at $T_{c}$. Such a dramatic change cannot be simply explained by the thermal occupation factors, as is usual for two-phonon processes in a harmonic system. The relative strength of the sideband in this case, as we mentioned above, is due to anharmonic coupling to the neighboring $\mathrm{CN}$ mode. Such a coupling, between a twophonon process and a one-phonon state, is usually referred to as Fermi resonance previously observed in other solids. ${ }^{16}$ To treat this resonance we take a quasiharmonic mode coupling analysis by neglecting the damping ${ }^{12}$ and assume that the phonon density of states does not change significantly with the temperature. With $\omega_{\mathrm{s}}$ and $\omega_{\mathrm{CN}}$ being the unperturbed frequency of the sideband and the $\mathrm{CN}$ mode, respectively, the new eigenfrequencies and eigenvectors, after allowing for the coupling, are determined by the following matrix equation:

$$
\left(\begin{array}{cc}
\omega_{\mathrm{s}} & V \\
V & \omega_{\mathrm{CN}}
\end{array}\right)\left(\begin{array}{l}
C_{\mathrm{s}, \mathrm{CN}}^{1} \\
C_{\mathrm{s}, \mathrm{CN}}^{2}
\end{array}\right)=\Omega_{\mathrm{s}, \mathrm{CN}}\left(\begin{array}{l}
C_{\mathrm{s}, \mathrm{CN}}^{1} \\
C_{\mathrm{s}, \mathrm{CN}}^{2}
\end{array}\right),
$$

where $\Omega_{\mathrm{s}, \mathrm{CN}}$ represents the renormalized frequencies of the two coupled modes, $V$ is the coupling constant, and $C_{\mathrm{s}, \mathrm{CN}}^{1}$ and $C_{\mathrm{s}, \mathrm{CN}}^{2}$ are the two coefficients describing the admixture of the two initial modes after the coupling. Equation (1) gives

$$
\Omega_{\mathrm{s}, \mathrm{CN}}=\frac{\left(\omega_{\mathrm{s}}+\omega_{\mathrm{CN}}\right)}{2} \pm \sqrt{\left(\frac{\omega_{\mathrm{s}}-\omega_{\mathrm{CN}}}{2}\right)^{2}+V^{2}},
$$

and the new eigenvectors $\left|\Omega_{\mathrm{s}, \mathrm{CN}}\right\rangle=C_{\mathrm{s}, \mathrm{CN}}^{1}\left|\omega_{\mathrm{s}}\right\rangle+C_{\mathrm{s}, \mathrm{CN}}^{2}\left|\omega_{\mathrm{CN}}\right\rangle$ are given by

$$
\begin{gathered}
C_{\mathrm{s}, \mathrm{CN}}^{1}=\frac{V}{\Omega_{\mathrm{s}, \mathrm{CN}}-\omega_{\mathrm{s}}} C_{\mathrm{s}, \mathrm{CN}}^{2}, \\
\left(C_{\mathrm{s}, \mathrm{CN}}^{1}\right)^{2}+\left(C_{\mathrm{s}, \mathrm{CN}}^{2}\right)^{2}=1 .
\end{gathered}
$$

If $\Delta \omega=\omega_{\mathrm{s}}-\omega_{\mathrm{CN}}$ and $\Delta \Omega=\Omega_{\mathrm{s}}-\Omega_{\mathrm{CN}}$ represent the frequency differences of the two modes before and after the perturbation, then Eq. (2) can be reduced to

$$
\Delta \omega=\sqrt{(\Delta \Omega)^{2}-4 V^{2}} .
$$

Assuming the Raman activity of the sideband is negligible $(\approx 0)$ compared to the $\mathrm{CN}$ internal mode before the pertur-
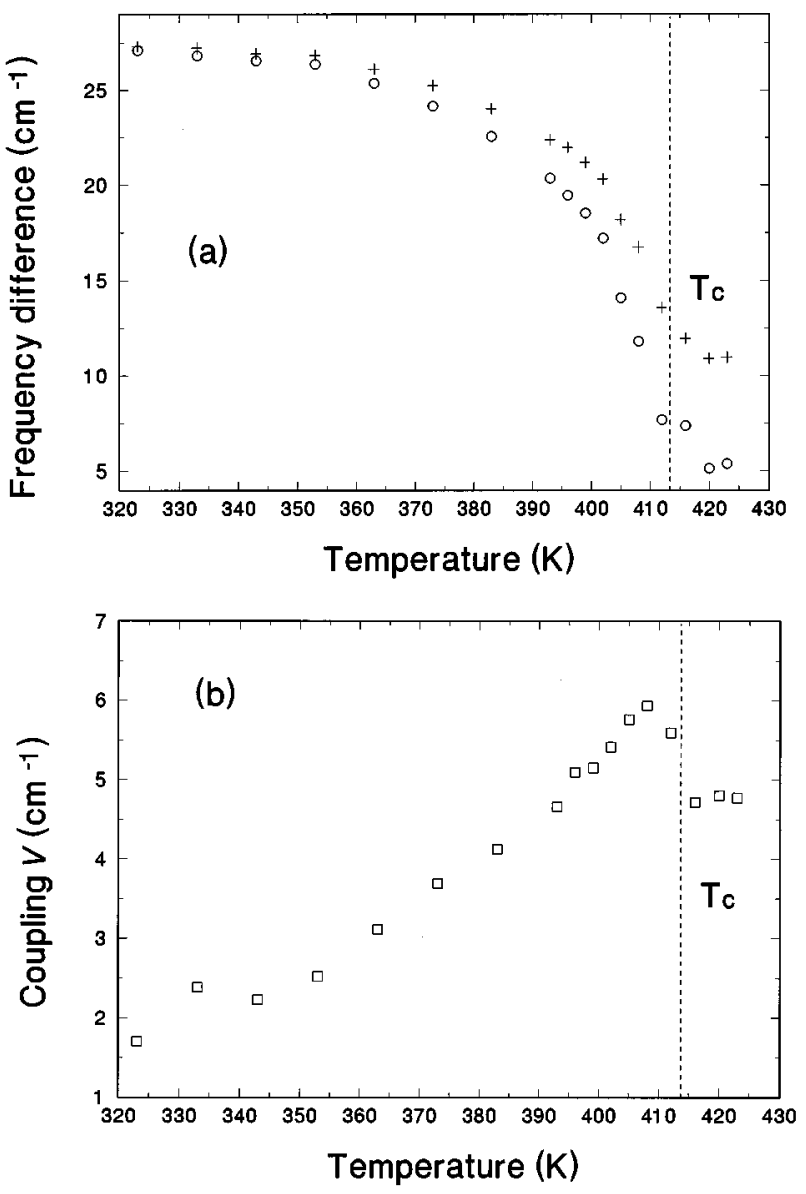

FIG. 3. The coupling between the $\mathrm{CN}$ mode and the sideband was estimated using a two coupled oscillator model. (a) The bare frequency difference $(O)$ obtained from this mode coupling calculation is plotted, together with the observed value $(+)$, against temperature. (b) shows the nearly linear temperature dependence found for the coupling parameter $V$.

bation, then the Raman intensity of the renormalized modes $I\left(\left|\Omega_{\mathrm{s}, \mathrm{CN}}\right\rangle\right)$ is proportional to the square of the eigenvector component of $\left|\omega_{\mathrm{CN}}\right\rangle$, i.e., $I\left(\left|\Omega_{\mathrm{s}, \mathrm{CN}}\right\rangle\right) \propto\left(C_{\mathrm{s}, \mathrm{CN}}^{1}\right)^{2}$. The ratio of these intensities can be calculated using Eqs. (2)-(4) as

$$
r=\frac{I\left(\left|\Omega_{\mathrm{s}}\right\rangle\right)}{I\left(\left|\Omega_{\mathrm{CN}}\right\rangle\right)}=\frac{V^{2}+\left(\Omega_{\mathrm{CN}}-\omega_{\mathrm{s}}\right)^{2}}{V^{2}+\left(\Omega_{\mathrm{s}}-\omega_{\mathrm{s}}\right)^{2}} .
$$

From Eqs. (4) and (5) the coupling $V$ then can be obtained

$$
V=\frac{\Delta \Omega \sqrt{r}}{1+r} .
$$

Note that both the ratio of intensity $r$ and the frequency difference $\Delta \Omega$ of the two renormalized modes can be deduced from the experimental data, and therefore, the bare frequency difference $\Delta \omega$ and the coupling $V$ can be obtained using Eqs. (4) and (6).

Using the experimentally determined values of $r$ and $\Delta \Omega$, we obtain the values of $\Delta \omega$ and $V$ and their temperature dependence which are plotted in Figs. 3(a) and 3(b). It can be seen that the bare frequency difference $\Delta \omega$ changes by more than $70 \%$ approaching $T_{c}$. Such a softening, about $20 \mathrm{~cm}^{-1}$, is far larger than that observed previously ${ }^{1,3,8,9}$ for any mode 
of KSCN. Also, the coupling $V$ shows a nearly linear increase with temperature up to $T_{c}$ with a slope of about $5.56 \times 10^{-2} \mathrm{~cm}^{-1} / \mathrm{K}$, and decreases after the phase transition. The magnitude of the coupling $V$ is comparable to the frequency shifts of the $\mathrm{CN}$ mode and the sideband. However, it should be noted that even at $T \sim T_{c} V$ is only about $0.25 \%$ of the frequency of the $\mathrm{CN}$ mode. As regards to the specific origin of this coupling, one possibility we would suggest, is that it is due to rotational-librational interaction in the $\mathrm{SCN}^{-}$ molecule. Specifically the CN stretching mode causes a fluctuation in the rotational moment of inertia. Thus, in the crystal, it affects the frequency of the $\mathrm{SCN}^{-}$librational motions involved in the various low-lying lattice modes. Hence the sideband and its dramatic softening probably reflects a strong decrease in the librational frequency, to the point that, at $T_{c}$, the librations transform to hindered $90^{\circ}$ rotations.

In summary, both the $\mathrm{CN}$ internal mode and the sideband were found to exhibit anomalous changes on approaching the order-disorder phase transition of KSCN. The sideband shows a substantial softening suggesting strong involvement of an off-zone-center lattice instability in the order-disorder transition. In addition, a quasiharmonic mode coupling model is employed to calculate the Fermi-resonance-type coupling between the $\mathrm{CN}$ mode and the sideband.

This work was supported by the U.S. Army Research Office under Grant No. DAAG-55-97-1-0106. We thank R. Kirby, W. N. Mei, R. Feile, and E. Sherman for helpful discussion.
${ }^{1}$ W. Schranz, Phase Transit. 51, 1 (1994).

${ }^{2}$ O. Blaschko, W. Schwarz, W. Schranz, and A. Fuith, Phys. Rev. B 44, 9159 (1991).

${ }^{3}$ Z. Iqbal, L. H. Sarma, and K. D. Möller, J. Chem. Phys. 57, 4728 (1972).

${ }^{4}$ P. da R. Andrade, A. D. Prasad Rao, R. S. Katiyer, and S. P. S. Porto, Solid State Commun. 12, 847 (1973).

${ }^{5}$ M. Sakiyama, H. Suga, and S. Seki, Bull. Chem. Soc. Jpn. 36, 1025 (1963).

${ }^{6}$ Nguyen Quy Dao and G. R. Wilkinson, J. Chem. Phys. 59, 1319 (1973).

${ }^{7}$ S. S. Ti, S. F. A. Kettle, and Ø. Ra, Spectrochim. Acta A 32, 1603 (1976).

${ }^{8}$ F. J. Owens, Solid State Commun. 29, 789 (1979).

${ }^{9}$ A. W. Hassan Ali and M. H. Talaat, Phys. Rev. B 43,
3549 (1991).

${ }^{10}$ S. Sathaiah and H. D. Bist, Z. Phys. B 84, 423 (1991).

${ }^{11}$ C. H. Wang and P. A. Fleury, in Light Scattering Spectra of Solids, edited by G. B. Wright (Spring-Verlag, New York, 1969), p. 651.

${ }^{12}$ J. F. Scott, Rev. Mod. Phys. 46, 83 (1974).

${ }^{13}$ A. Fuith, T. Streuselberger, and H. Warhanek, J. Cryst. Growth 97, 469 (1989).

${ }^{14}$ S. S. Ti and Ø. Ra, J. Chem. Phys. 73, 5738 (1980).

${ }^{15}$ M. Cardona, in Light Scattering in Solids (Ref. 11), p. 19.

${ }^{16}$ J. F. Scott, Phys. Rev. Lett. 21, 907 (1968); recently this type of resonance has been observed in high-pressure infrared spectra of compressed $\mathrm{H}_{2} \mathrm{O}$ and $\mathrm{D}_{2} \mathrm{O}$ ices by V. V. Struzhkin, A. F. Goncharov, R. J. Hemley, and H. K. Mao, Phys. Rev. Lett. 78, 4446 (1997). 